the secretion of C-reactive protein (CRP) by an automated analyzer.

Results IL-17 and TNF $\alpha$ induced in synergy the hepatic IL-6 production $(>8$ fold, $\mathrm{p}<0.01)$ and CCL-20 expression ( $>100$ fold, $\mathrm{p}<0.01$ ). CRP secretion was induced by IL-17/ TNF $\alpha$ and the anti-IL-6R inhibited this induction. CCL-20 expression was not increased by IL-6 stimulation or reduced by the addition of the anti-IL-6R to the IL-17/TNF $\alpha$ treatment. In HepaRG cell-PBMC cultures, IL-6 and IL-17 were produced only in PHA-activated conditions and the IL-6 and IL-17 levels were higher in co-cultures vs PBMC monocultures $(>14$ and $>2$ fold respectively, $\mathrm{p}<0.01)$. Transwell system that avoids direct cell-cell contact decreased the IL-6 and IL17 secretion by 4- and 2-fold respectively in PHA-activated HepaRG cell-PBMC cultures. CRP production increased when $\mathrm{PHH}$ were cultured in presence of PHA-activated PBMC compared to $\mathrm{PHH}$ alone with PHA.

Conclusions The IL-17/TNF $\alpha$ synergistic effect on hepatocytes mediates systemic inflammation by inducing CRP secretion through the IL-6 pathway and mononuclear cell recruitment by acting on CCL-20 expression in an IL- 6 independent manner. Direct and indirect hepatocyte-PBMC interactions contribute to the hepatic inflammatory response by increasing the IL6 and IL-17 secretion. The induction of Th17 cell differentiation by IL- 6 and the increase of CCL- 20 mediated Th17 cell recruitment by IL-17 may lead to a vicious and chronic inflammatory cycle.

Disclosure of interest None declared

\section{P078 A GENETIC VARIANT OF IL-32 IS ASSOCIATED WITH THE EX VIVO CYTOKINE PRODUCTION OF ANTI-TNF TREATED PBMCS ISOLATED FROM RHEUMATOID ARTHRITIS PATIENTS}

\footnotetext{
${ }^{1,2} \mathrm{C}$ Popa* ${ }^{3} \mathrm{M}$ Damen, ${ }^{3} \mathrm{~K}$ Schraa, ${ }^{2} \mathrm{~L}$ Tweehuysen, ${ }^{2} \mathrm{~A}$ den Broeder, ${ }^{3} \mathrm{M}$ Netea, ${ }^{3} \mathrm{~L}$ Joosten. ${ }^{1}$ Rheumatology, Radboud UMC; ${ }^{2}$ Rheumatology, Sint Maartenskliniek; ${ }^{3}$ Internal Medicine, Radboud UMC, Nijmegen, Netherlands
}

\subsection{6/annrheumdis-2018-EWRR2018.95}

Introduction Since the introduction of biologics in the treatment of rheumatoid arthritis (RA) disease outcome improved. Still, about $40 \%$ of RA patients do not respond to therapy with TNF $\alpha$ blockers. Previously, a strong link between TNF $\alpha$ and interleukin (IL) -32 has been reported in RA. ${ }^{1}$

Objectives We hypothesise that a promoter single nucleotide polymorphism (SNP) in IL-32 can affect clinical responsiveness to anti-TNF $\alpha$ treatment in RA patients, functioning as a new biomarker in treatment of RA. ${ }^{2}$

Methods Peripheral mononuclear cells (PBMCs) from RA patients and healthy individuals were stimulated with RPMI or recombinant human (rh)TNF $\alpha$ to study the mRNA and protein expression of IL-32 and other pro-inflammatory cytokines. Moreover, disease activity scores (DAS28), 'in vitro response' and clinical response to anti-TNF $\alpha$ therapy (etanercept, adalimumab), of RA patients were measured and all were stratified for the IL-32 SNP (C/T).

Results Stimulation of PBMCs from RA patients was followed by higher IL-32 protein production and a tendency towards higher IL-32 $\beta$ and IL-32 $\gamma$ mRNA expression compared to healthy individuals. When data was stratified for the IL-32 promoter SNP, patients bearing the CC genotype showed higher IL-32 protein expression. Of interest, these patients also produced more cytokines. Even though the DAS28 did not depend on the presence of the promoter SNP, the 'ex vivo' cytokine response did have a different pattern in clinical responders depending on the genotype.

Conclusions IL-32 mRNA and protein production was higher in RA patients compared to healthy individuals, with a trend towards higher concentrations in patients bearing the CC genotype. Regardless of the fact that the promoter SNP was not associated with disease activity, IL-1 beta production in the CC-genotype might predict clinical response to either etanercept or adalimumab.

\section{REFERENCES}

1. Heinhuis B, et al. Ann Rheum Dis 2011;70:660-7.

2. Damen MS, et al. Sci Rep 2017;7:41629.

Disclosure of interest None declared

\section{P079 INTERLEUKIN-27 REGULATES THE MAGNITUDE OF THE ECTOPIC GERMINAL CENTRE RESPONSE IN A VIRAL- INDUCIBLE MODEL OF SIALADENITIS}

${ }^{1} \mathrm{D}$ Lucchesi*, ${ }^{1} \mathrm{E}$ Pontarini, ${ }^{1} \mathrm{R}$ Coleby, ${ }^{2} \mathrm{GW}$ Jones, ${ }^{2} \mathrm{DG}$ Hill, ${ }^{1} \mathrm{C}$ Pitzalis, ${ }^{1} \mathrm{M}$ Bombardieri. ${ }^{1}$ Experimental Medicine and Rheumatology, Queen Mary University of London, London; ${ }^{2}$ Division of Infection and Immunity, School of Medicine, Cardiff University, Cardiff, UK

\subsection{6/annrheumdis-2018-EWRR2018.96}

Introduction Ectopic lymphoid structures (ELS) are leukocytes aggregates that form in tissues affected by chronic inflammation. In autoimmunity, ELS play an active role in disease progression and are typically associated with aggressive disease. Thus, understanding the mechanisms that trigger ELS formation is of paramount importance for therapeutic targeting of this process. Interleukin-27 (IL-27) is prominently associated with the negative control of adaptive immunity, and in particular in the suppression of Th17-type responses.

Objectives To elucidate the role of IL-27 in the control of lymphoid neogenesis and its functional relationship with aberrant IL-17 production in a murine model of inducible ELS, where the local administration of a replication-deficient adenovirus (AdV) triggers the formation of ectopic germinal centres in salivary glands (SG).

Methods A single administration of AdV was delivered by cannulation directly into the SG of wild-type (WT) and IL-27Rdeficient $\left(I l 27 \mathrm{ra}^{-/}\right)$mice. For IL-17A blockade, an anti-mouse IL-17A antibody or IgG control was administered systemically. ELS development and peripheral immune responses were temporally tracked by immuno-histopathology, flow cytometry, and real-time qPCR.

Results AdV cannulation induced an early upregulation of IL27 and IL-27R in WT mice SG, which was mirrored by an increase in the infiltration of IL-27-producing T, B and NK cells. AdV-challenged $\mathrm{Il}_{2} 7 \mathrm{ra}^{-/-}$mice developed exacerbated salivary gland inflammation, and by day- 19 post $\mathrm{AdV}$ challenge developed larger and more abundant ELS as compared to WT mice. Moreover, $\mathrm{Il}_{2} 7 \mathrm{ra}^{-/}$mice displayed a heightened expression of homeostatic cytokines, chemokines and their corresponding receptors that are required for lymphoid neogenesis (e.g., Cxcl13, Ccl19 and Ltb). IL-27R-deficient mice also displayed elevated markers of functional germinal centre responses (e.g., activation-induced deaminase, AID). Underpinning the exaggerated development of ELS in $\mathrm{Il}_{2} 7 \mathrm{ra}^{-/}$mice was the preferential expansion of IL-17-producing $\mathrm{T}$ helper (Th)17 cells, which was linked to a reduction in the Th1 cell 